\title{
A novel strategy for searching for $C P$ violations in the baryon sector
}

\author{
Zhen-Hua Zhang ${ }^{a}$ and Xin-Heng Guo ${ }^{b}$ \\ ${ }^{a}$ School of Nuclear Science and Technology, University of South China, \\ Hengyang, Hunan, 421001, China \\ ${ }^{b}$ College of Nuclear Science and Technology, Beijing Normal University, \\ Beijing, 100875, China \\ E-mail: zhangzh@usc.edu.cn, xhguo@bnu.edu.cn
}

ABstract: Despite the large baryon-anti-baryon asymmetry in the observable Universe, the closely related phenomenon - the violation of the combined charge and parity symmetry $(C P \mathrm{~V})$ - has not been observed in the baryon sector in laboratories. In this paper, a new strategy for searching for $C P \mathrm{~V}$ in heavy hadron multi-body decays is proposed, in which a set of novel observables measuring $C P \mathrm{~V}$ in such decays - the partial wave $C P$ asymmetries (PWCPAs) - are introduced. This strategy is model-independent and applicable to multi-body decays of heavy hadrons with arbitrary spin configurations in both initial and final states, and with any number of particles in the final state. It is especially applicable for $C P \mathrm{~V}$ investigations in multi-body decays of heavy baryons. As applications of this strategy, we suggest to measure the PWCPAs in some decay channels of bottom baryons such as $\Lambda_{b}^{0} \rightarrow p \pi^{-} \pi^{+} \pi^{-}, \Lambda_{b} \rightarrow p K^{-} \pi^{+} \pi^{-}, \Lambda_{b}^{0} \rightarrow p \pi^{-} K^{+} K^{-}, \Lambda_{b}^{0} \rightarrow \Lambda K^{+} \pi^{-}$, and $\Lambda_{b}^{0} \rightarrow p \pi^{-} K_{s}$.

Keywords: CP violation, Discrete Symmetries, Heavy Quark Physics

ArXiv EPrint: 2103.11335 


\section{Contents}

1 Introduction 1

2 Partial Wave $C P$ Asymmetries 2

3 Contribution of the interference of nearby resonances to PWCPA: underlining dynamics and Selection Rules 4

4 Applications to baryon decays $\quad 6$

5 Conclusion $\quad 12$

\section{Introduction}

The violation of the symmetry of the combined charge-parity $(C P)$ transformation, as a phenomenon closely related to the matter-antimatter asymmetry in the Universe [1], was first discovered in the neutral kaon system [2], and is accommodated in the Standard Model (SM) of particle physics by the Cabibbo-Kobayashi-Maskawa (CKM) mechanism that describes the transitions between up- and down-type quarks [3, 4]. $C P$ violation $(C P \mathrm{~V})$ in hadron decays is described by the asymmetry between the particle and antiparticle decay rates, which has been observed in many decay channels of heavy mesons [5-9]. On the other hand, despite the large baryon-anti-baryon asymmetry in the observable Universe, no $C P \mathrm{~V}$ has ever been observed in the baryon sector in laboratories [10-15].

One reason for the negative result in searching for $C P \mathrm{Vs}$ in the baryon sector is the substantially lower statistics compared with the meson case. One of the latest examples for the $C P \mathrm{~V}$ measurements in bottom baryon decays can be found in ref. [16], from which one can clearly see that the statistics for the bottom baryon case is indeed much lower than that in the bottom meson case (see, for example, ref. [17]). So although $C P$ asymmetries (CPAs) of a few or several tens of percent is expected in some decay channels of heavy baryons [18], no significant evidence of $C P \mathrm{~V}$ has yet been found experimentally in the baryon sector.

The rich resonance structures in multi-body decays of heavy hadrons provide us opportunities for $C P \mathrm{~V}$ searching in these decay channels, as the interference between different intermediate resonances may result in large regional $C P A s(\mathrm{rCPAs})$ distributed in the phase space, although the overall CPA may be small due to cancellations among different parts of the phase space. In fact, it has been observed that for some three-body decay channels of $B$ mesons such as $B^{+} \rightarrow \pi^{+} \pi^{-} \pi^{+}$and $B^{+} \rightarrow K^{+} \pi^{-} \pi^{+}$, very large rCPAs are localized in certain small parts of the phase space, thanks to the relatively larger statistics [8]. Some of these $\mathrm{r} C P$ As can indeed be explained by the interference between a resonance and another 
one nearby via the introduction of a non-perturbative relative strong phase between their corresponding amplitudes [19-21]. Large $\mathrm{r} C P$ As associated with the interference between close resonances are also expected in multi-body decays of heavy baryons. However, the aforementioned low statistics problem leads to the direct measurements of the $\mathrm{r} C P$ As for multi-body decays of heavy baryons suffering from large uncertainties and hence no $C P \mathrm{~V}$ has been established in heavy baryon decays. Even though one can partly fix the low statistic issue by merging small bins in the phase space region into larger ones, the rCPAs of the enlarged bins are usually much smaller since there are almost always cancellations among these aforementioned small bins when obtaining the $\mathrm{rCPAs}$ of the enlarged bins. Therefore, besides the direct measurements of the rCPAs or the overall CPAs, a more efficient and systematic method for $C P \mathrm{~V}$ hunting is thus urgent for multi-body decays of heavy baryons.

Motivated by the above discussion, in this paper, we will introduce a set of new $C P \mathrm{~V}$ observables - the Partial Wave CP Asymmetries - associated with the intermediate resonances in the multi-body decays of heavy hadrons. It will be shown that the Partial Wave $C P$ Asymmetries can make use of the data in a more efficient way, and thus, can potentially overcome the low statistics problem associated with the heavy baryon decays. It is possible that with this new strategy, the establishment of $C P \mathrm{~V}$ in the baryon sector may be hastened.

This paper is organized as follows. In section 2, we introduce the set of observables, the Partial Wave $C P$ Asymmetries, and discuss its measurement method and its advantages comparing with other observables or techniques for $C P \mathrm{~V}$ measurements. In section 3 , we focus mainly on the situation of the interference of nearby resonances, in which the nonperturbative effect of strong interaction could amplify the CPV signal. We briefly discuss the correlation of the underline dynamics for $C P \mathrm{~V}$ with this newly introduced observables for this situation. An set of important Selection Rules which tell weather the interference effect are present or absent in certain Partial Wave $C P$ Asymmetries are also presented. In section 4, we apply the newly introduced observables to the four-body decay process, $\Lambda_{b}^{0} \rightarrow p \pi^{-} \pi^{+} \pi^{-}$. In the last section, we briefly give the conclusion.

\section{Partial Wave $C P$ Asymmetries}

Without lose of generality, consider a multi-body decay, $H \rightarrow h_{1} h_{2} h_{3} \cdots h_{n}$, where $H$ is a heavy hadron, while $h_{k}(k=1,2,3, \cdots, n)$ are light ones. We will focus on the region of the phase space where an intermediate resonance $R_{a}$ dominates, i.e., the invariant mass squared of the $h_{1} h_{2}$ system, $s_{12}$, lies between $\left(m_{R_{a}}-\alpha_{R_{a}}\right)^{2}$ and $\left(m_{R_{a}}+\beta_{R_{a}}\right)^{2}$, with $m_{R_{a}}$ the mass of $R_{a}$, and $\alpha_{R_{a}}$ and $\beta_{R_{a}}$ of the same other as the decay width of $R_{a}$. Then, this multi-body decay will be dominated by the cascade decay $H \rightarrow R_{a} h_{3} \cdots h_{n}, R_{a} \rightarrow h_{1} h_{2}$. Assuming that the initial particle $H$ is produced unpolarized, the differential decay width can then be expressed as

$$
d \Gamma \propto \overline{|\mathcal{M}|^{2}} d c_{\theta_{1}^{*}}
$$

after summing over the helicities (or the z-components of the spins) of both the initial and the final particles, and integrating over the phase space variables $-s_{12}$ is integrated from 
$\left(m_{R_{a}}-\alpha_{R_{a}}\right)^{2}$ to $\left(m_{R_{a}}+\beta_{R_{a}}\right)^{2}$ for the phase space integration- except $c_{\theta_{1}^{*}}$, where $\theta_{1}^{*}$ is the relative angle between the momentum of $h_{1}$ and that of $H$ in the rest frame of the $h_{1} h_{2}$ system, and $c_{\theta_{1}^{*}}$ is its cosine, $\overline{|\mathcal{M}|^{2}}$ is the spin-averaged and phase space-integrated (except $c_{\theta_{1}^{*}}$ ) square of the decay amplitude, which can be further expanded with the aid of the Legendre's polynomials as

$$
\overline{|\mathcal{M}|^{2}} \propto \sum_{j=0}^{\infty} w^{(j)} P_{j}\left(c_{\theta_{1}^{*}}\right)
$$

where the coefficients $w^{(j)}$ can be expressed as

$$
w^{(j)} \propto \int_{-1}^{1} \overline{|\mathcal{M}|^{2}} P_{j} d c_{\theta_{1}^{*}}
$$

according to the orthogonality properties of $P_{j}$ s. Since this decay process is a weak one, $C P$ V may show up as the difference between $w^{(j)}$ and $\bar{w}^{(j)}$, where $\bar{w}^{(j)}$ corresponds to $w^{(j)}$ for the $C P$ conjugate process $\bar{H} \rightarrow \bar{h}_{1} \bar{h}_{2} \bar{h}_{3} \cdots \bar{h}_{n}$. One can then introduce a set of observables, which will be called as the Partial Wave CP Asymmetries (PWCPAs), and for the $j$ th-wave:

$$
A_{C P}^{(j)} \equiv \frac{w^{(j)}-\bar{w}^{(j)}}{w^{(j)}+\bar{w}^{(j)}} .
$$

The PWCPAs are experiment-friendly observables, which can be extracted easily by fitting the data with eq. (2.2). Another equivalent but more straightforward method is described as follows. According to the orthogonality property of $P_{j}$ one can see that different events collected in the data do not contribute to $w^{(j)}$ equally. Instead, they contribute with a weight which is proportional to $P_{j}\left(c_{\theta_{1}^{*}}\right)$. For an event labeled as $k$, one can easily determine its corresponding $c_{\theta_{1}^{*}}$, which will be denoted as $c_{\theta_{1, k}^{*}}$. Accordingly, one can introduce the so called $P_{j}$-weighted event yield,

$$
\mathcal{N}_{j-\text { weighted }} \equiv \sum_{k} P_{j}\left(c_{\theta_{1, k}^{*}}\right),
$$

which is proportional to $w^{(j)}{ }^{1}$ The $P_{j}$-weighted event yield for the $C P$ conjugate process $\overline{\mathcal{N}}_{j \text {-weighted }}$ can be obtained in the same way. With the above $P_{j}$-weighted event yields $\mathcal{N}_{j-\text { weighted }}$ and $\overline{\mathcal{N}}_{j \text {-weighted }}$, one can easily obtain the experimental values of the PWCPAs through

$$
A_{C P}^{(j), \exp }=\frac{\mathcal{N}_{j-\text { weighted }}-\overline{\mathcal{N}}_{j \text {-weighted }}}{\mathcal{N}_{j \text {-weighted }}+\overline{\mathcal{N}}_{j \text {-weighted }}} .
$$

From the measurement methods proposed above, one can see that with the newly introduced observables PWCPAs one can make use of the data in a more efficient way,

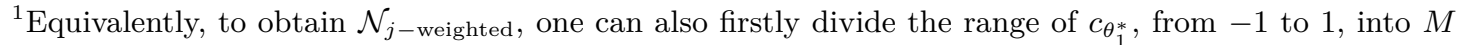
small intervals, with each interval corresponding to an average value of $c_{\theta_{1}^{*}}$, which will be denoted as $c_{\theta_{1, m}^{*}}$, $(m=1,2, \cdots, M)$. Secondly, count the event number in each interval, which will be denoted as $N_{m}$ for the interval $m$. Then, one can easily see that the $P_{j}$-weighted event yields $\mathcal{N}_{j \text {-weighted }}$ can be expressed as $\mathcal{N}_{j \text {-weighted }}=\sum_{m} N_{m} P_{j}\left(c_{\theta_{1, m}^{*}}\right)$. This method will be used to obtain the PWCPA in the simulation in section 4 .
} 
comparing with the r $C P$ As which are widely measured in multi-body decays of $B$ mesons currentle. This is because instead of dividing the value range of $c_{\theta_{1}^{*}}$ into small bins and making use of only the events in each bins when measuring the rCPAs, each of the PWCPAs makes use of all the events distributed in the whole range of $c_{\theta_{1}^{*}}$ from -1 to 1 . This can be seen clearly when the integration over $c_{\theta_{1}^{*}}$ from -1 to 1 is performed based on the orthogonality properties of $P_{j}$, indicating the usage of the events over the whole range of $c_{\theta_{1}^{*}}$. This can also be seen in more detail from the measurement method proposed above. Making use of all the events distributed in the whole range of $c_{\theta_{1}^{*}}$ is very important when statistics is not large enough, as in the case of heavy baryon decays. Another important feature of the PWCPAs which can be seen from the above discussion is that, they provide a more efficient model-independent approach for hunting for $C P \mathrm{~V}$ than the currently used amplitude analysis technique [22] in multi-body decays of heavy hadrons, in which model dependence could potentially arise [23].

\section{Contribution of the interference of nearby resonances to PWCPA: underlining dynamics and Selection Rules}

The PWCPAs $A_{C P}^{(j)}$ are in fact $C P \mathrm{~V}$ observables for the decay $H \rightarrow R_{a} h_{3} \cdots h_{n}$, if $R_{a}$ is the only dominate resonance in the phase space region, $\left(m_{R_{a}}-\alpha_{R_{a}}\right)^{2}<s_{12}<\left(m_{R_{a}}+\beta_{R_{a}}\right)^{2}$. For example, $A_{C P}^{(0)}$ is nothing but the $C P$ A parameter for the decay $H \rightarrow R_{a} h_{3} \cdots h_{n}{ }^{2}$ However, $C P \mathrm{~V}$ is usually relatively small in the single-resonance-dominance situation. Hence, interference with a second resonance is required to generate larger $C P \mathrm{~V}$. In this situation, the decay amplitude can then be approximated by the sum of the two cascade decays, $H \rightarrow R_{a} h_{3} \cdots h_{n}, R_{a} \rightarrow h_{1} h_{2}$, and $H \rightarrow R_{b} h_{3} \cdots h_{n}, R_{b} \rightarrow h_{1} h_{2}$, in the phase space region $\left(m_{R_{a}}-\alpha_{R_{a}}\right)^{2}<s_{12}<\left(m_{R_{a}}+\beta_{R_{a}}\right)^{2}$. In this situation, the PWCPAs are no longer just $C P V$ observables for $H \rightarrow R_{a} h_{3} \cdots h_{n}$. Instead, they will also contain contribution from the resonance $R_{b}$, and moreover, that from the interference between the two resonances $R_{a}$ and $R_{b}$. The interference of nearby resonances has great impact on $C P \mathrm{~V}$ in multi-body decays of bottom hadrons, either through rCPAs, as has been observed in some threebody decays of $B$ meson, and/or through PWCPAs, which will be seen in more details in what follows.

It is crucially important to find out the correlation between the origin of $C P \mathrm{~V}$ in the PWCPAs, $A_{C P}^{(j)}$, and the underlining dynamics. To achieve this, one needs to substitute the decay amplitudes into $\overline{|\mathcal{M}|^{2}}$. After some algebra, one arrive at the expression of $w^{(j)}$ as

$$
w^{(j)}=\left\langle\frac{\mathcal{S}_{a a}^{j} \mathcal{W}_{a a}^{j}}{\left|s_{R_{a}}\right|^{2}}\right\rangle+\left\langle\frac{\mathcal{S}_{b b}^{j} \mathcal{W}_{b b}^{j}}{\left|s_{R_{b}}\right|^{2}}\right\rangle+\left\langle 2 \Re\left(\frac{\mathcal{S}_{a b}^{j} \mathcal{W}_{a b}^{j}}{s_{a} s_{b}^{*}}\right)\right\rangle
$$

where $s_{R_{a} / R_{b}}$ are the denominators of the Breit-Wigner propagators and take the form $s_{R_{a} / R_{b}}=s_{12}-m_{R_{a} / R_{b}}^{2}+i m_{R_{a} / R_{b}} \Gamma_{R_{a} / R_{b}}$, the notation " $\langle\cdots\rangle$ " represents the integral over

\footnotetext{
${ }^{2}$ Of course, $A_{C P}^{(0)}$ is also the $\mathrm{r} C P \mathrm{~A}$ for the whole $R_{a}$-dominated phase space region, $\left(m_{R_{a}}-\alpha_{R_{a}}\right)^{2}<$ $s_{12}<\left(m_{R_{a}}+\beta_{R_{a}}\right)^{2}$.
} 
the phase space except $c_{\theta_{1}^{*}}$, and

$$
\begin{aligned}
\mathcal{W}_{X Y}^{j} & =\sum_{\sigma}(-)^{\sigma-j}\left\langle j_{X} j_{Y}-\sigma \sigma \mid j 0\right\rangle \sum_{m_{z} \lambda_{3} \cdots} \mathcal{M}_{\sigma \lambda_{3} \cdots}^{J m_{z}, X} \mathcal{M}_{\sigma \lambda_{3} \cdots}^{J m_{z}, Y *}, \\
\mathcal{S}_{X Y}^{j} & =\left.\sum_{\lambda_{1}^{\prime} \lambda_{2}^{\prime}}(-)^{j-\lambda^{\prime}}\left\langle j_{X} j_{Y}-\lambda^{\prime} \lambda^{\prime} \mid j 0\right\rangle \mathcal{G}_{\lambda_{1}^{\prime} \lambda_{2}^{\prime}}^{j \mathcal{G}_{X}} \mathcal{G}_{\lambda_{1}^{\prime} \lambda_{2}^{\prime}}^{\left.j\right|^{*}}\right|_{\lambda^{\prime}=\lambda_{1}^{\prime}-\lambda_{2}^{\prime}},
\end{aligned}
$$

where $X, Y=a, b, j_{a / b}$ are the spins of $R_{a / b}, \mathcal{M}_{\sigma \lambda_{3} \cdots}^{J m_{z}, a / b}$ are the covariant decay amplitudes for the weak decays $H \rightarrow R_{a / b} h_{3} \cdots h_{n}$ with $\sigma, \lambda_{3}, \cdots$ being the helicities of $R_{a / b}, h_{3}, \cdots$ defined in the rest frame of $H$, respectively, $\mathcal{G}_{\lambda_{1}^{\prime} \lambda_{2}^{\prime}}^{j_{a / b}}$ are the helicity decay amplitudes [24] for the strong decay processes $R_{a / b} \rightarrow h_{1} h_{2}$ in the rest frame of the $h_{1} h_{2}$ system with $\lambda_{1(2)}^{\prime}$ being the helicities of $h_{1(2)}$ in the same frame, and the notation $\langle\cdots \mid \cdots\rangle$ 's are the Clebsch-Gordan coefficients. Among the three terms in eq. (3.1), the first two represent the contributions from the resonances $R_{a}$ and $R_{b}$ alone, respectively, while the last one represents the effect of the interference between $R_{a}$ and $R_{b}$.

As of aforementioned, the PWCPAs get their contributions from the difference between $w^{j}$ and $\bar{w}^{j}$. This difference is originated from the interference of amplitudes with different weak and strong phases. For example, for the case of bottom hadron decay, each of the weak decay amplitudes $\mathcal{M}_{\sigma \lambda_{3} \cdots}^{J m_{z}, a / b}$ can be further divided into a tree and penguin amplitudes, $\mathcal{M}_{\sigma \lambda_{3}, \cdots}^{J m_{z}, a / b}=\mathcal{M}_{\sigma \lambda_{3} \cdots}^{J m_{z}, a / b, \text { tree }}+\mathcal{M}_{\sigma \lambda_{3} \cdots}^{J m_{z}, a / b \text {,penguin }}$. All the three terms in eq. (3.1) could contribute to the PWCPAs. The contributions to the PWCPAs of the first two terms in eq. (3.1) come from the weak decay process $H \rightarrow R_{a / b} h_{3} \cdots h_{n}$ alone, i.e., the interference of the tree and penguin amplitudes for the decay via the same resonances $R_{a}$ or $R_{b}$. These two terms are proportional to the sine of the strong phase difference between the amplitudes of the tree and penguin operators for the same resonances $R_{a}$ or $R_{b}$, respectively, which is usually small unless some particular mechanism enters to generate a large phase difference. ${ }^{3}$ On the other hand, the last term in eq. (3.1) represents the interference between the two resonances $R_{a}$ and $R_{b}$. Its contribution to the PWCPAs is proportional to the sine of the strong phase difference between the amplitude through the resonance $R_{a}$ and that through $R_{b}$, i.e., tree and penguin amplitudes from different resonances. The strong phase difference between different resonances could be large because of the nonperturbative strong interaction effects, permitting the existence of large $\mathrm{r} C P A$ s and/or PWCPAs. In fact, large r $C P A s$ which are correlated with the aforementioned interference of different resonances have been observed in some three-body decays of $B$ meson. Similarly, the presence of the last term in $w^{j}$ in eq. (3.1) indicates that it can contribute to the PWCPAs, resulting in the PWCPAs which are large enough to be potentially detectable.

\footnotetext{
${ }^{3}$ Note that if there were only one resonance $R_{a}$ which dominates, $w^{j}$ would only contain the first term. In this situation, the PWCPAs are reduced to

$$
A_{C P}^{(j)}=\frac{\mathcal{W}_{a a}^{j}-\overline{\mathcal{W}}_{a a}^{j}}{\mathcal{W}_{a a}^{j}+\overline{\mathcal{W}}_{a a}^{j}},
$$

from which one can see that the PWCPAs are indeed observables for the decay $H \rightarrow R_{a} h_{3} \cdots h_{n}$ and they contain only the weak decay amplitudes $\mathcal{M}_{\sigma \lambda_{3} \cdots}^{J m_{z}, a}$.
} 


\begin{tabular}{|c|c|c|c|c|c|}
\hline$\left(J^{P} R_{a}, J^{P} R_{b}\right)$ & non-int. $j$ & int. $j$ & $\left(J^{P} R_{a}, J^{P} R_{b}\right)$ & non-int. $j$ & int. $j$ \\
\hline$\left(0^{+}, 0^{+}\right)$or $\left(0^{-}, 0^{-}\right)$ & 0 & 0 & $\left(\frac{1}{2}^{+}, \frac{1}{2}^{+}\right)$or $\left(\frac{1}{2}^{-}, \frac{1}{2}^{-}\right)$ & 0 & 0 \\
\hline$\left(0^{+}, 0^{-}\right)$or $\left(0^{-}, 0^{+}\right)$ & 0 & none & $\left(\frac{1}{2}^{+}, \frac{1}{}^{-}\right)$or $\left(\frac{1}{2}^{-}, \frac{1}{2}^{+}\right)$ & 0 & 1 \\
\hline$\left(0^{+}, 1^{+}\right)$or $\left(0^{-}, 1^{-}\right)$ & 0,2 & none & $\left(\frac{1}{2}^{+}, \frac{3}{2}^{+}\right)$or $\left(\frac{1}{2}^{-}, \frac{3}{2}^{-}\right)$ & 0,2 & 2 \\
\hline$\left(0^{-}, 1^{+}\right)$or $\left(0^{+}, 1^{-}\right)$ & 0,2 & 1 & $\left(\frac{1}{2}^{-}, \frac{3}{2}^{+}\right)$or $\left(\frac{1}{2}^{+}, \frac{3}{2}^{-}\right)$ & 0,2 & 1 \\
\hline$\left(0^{+}, 2^{+}\right)$or $\left(0^{-}, 2^{-}\right)$ & $0,2,4$ & 2 & $\left(\frac{1}{2}^{+}, \frac{5}{}^{+}\right)$or $\left(\frac{1}{2}^{-}, \frac{5}{2}^{-}\right)$ & $0,2,4$ & 2 \\
\hline$\left(0^{-}, 2^{+}\right)$or $\left(0^{+}, 2^{-}\right)$ & $0,2,4$ & none & $\left(\frac{1}{2}^{-}, \frac{5}{2}^{+}\right)$or $\left(\frac{1}{2}^{+}, \frac{5}{2}^{-}\right)$ & $0,2,4$ & 3 \\
\hline$\left(1^{+}, 1^{+}\right)$or $\left(1^{-}, 1^{-}\right)$ & 0,2 & 0,2 & $\left(\frac{3}{2}^{+}, \frac{3}{2}^{+}\right)$or $\left(\frac{3}{2}^{-}, \frac{3}{2}^{-}\right)$ & 0,2 & 0,2 \\
\hline$\left(1^{-}, 1^{+}\right)$or $\left(1^{+}, 1^{-}\right)$ & 0,2 & 1 & $\left(\frac{3}{2}^{-}, \frac{3}{2}^{+}\right)$or $\left(\frac{3}{2}^{+}, \frac{3}{2}^{-}\right)$ & 0,2 & 1,3 \\
\hline$\left(1^{+}, 2^{+}\right)$or $\left(1^{-}, 2^{-}\right)$ & $0,2,4$ & 2 & $\left(\frac{3}{2}^{+}, \frac{5}{2}^{+}\right)$or $\left(\frac{3}{2}^{-}, \frac{5}{2}^{-}\right)$ & $0,2,4$ & 2,4 \\
\hline$\left(1^{-}, 2^{+}\right)$or $\left(1^{+}, 2^{-}\right)$ & $0,2,4$ & 1,3 & $\left(\frac{3}{2}^{-}, \frac{5}{2}^{+}\right)$or $\left(\frac{3}{2}^{+}, \frac{5}{2}^{-}\right)$ & $0,2,4$ & 1,3 \\
\hline$\left(2^{+}, 2^{+}\right)$or $\left(2^{-}, 2^{-}\right)$ & $0,2,4$ & $0,2,4$ & $\left(\frac{5}{2}^{+}, \frac{5}{2}^{+}\right)$or $\left(\frac{5}{2}^{-}, \frac{5}{2}^{-}\right)$ & $0,2,4$ & $0,2,4$ \\
\hline$\left(2^{-}, 2^{+}\right)$or $\left(2^{+}, 2^{-}\right)$ & $0,2,4$ & 1,3 & $\left(\frac{5}{2}^{-}, \frac{5}{2}^{+}\right)$or $\left(\frac{5}{2}^{+}, \frac{5}{2}^{-}\right)$ & $0,2,4$ & $1,3,5$ \\
\hline
\end{tabular}

Table 1. The values of $j$ for which the non-interference and interference terms appear in $w^{(j)}$ and $\bar{w}^{(j)}$ according to SRs in the cases of integer and half-integer spins of $R_{a}$ and $R_{b}$.

With the aid of the properties of the Clebsch-Gordan coefficients and the parityconservation requirement for the processes $R_{a / b} \rightarrow h_{1} h_{2}$, it is easy to show that the interference and non-interference terms satisfy the following Selection Rules (SRs): the interference terms show up only when 1) $j=\left|j_{a}-j_{b}\right|, \cdots, j_{a}+j_{b}$ and 2) $(-)^{j} \pi_{a} \pi_{b}$ is positive, where $\pi_{a}$ and $\pi_{b}$ are the parities of $R_{a}$ and $R_{b}$, respectively; while the non-interference terms show up only when 1) $j$ is even, and 2) $j=0, \cdots, 2 j_{a / b}$. In table 1 , we list the values of $j$ for the interference and non-interference terms appear in $w^{(j)}$ and $\bar{w}^{(j)}$ according to the SRs for some spin-parity configurations of the resonances $R_{a}$ and $R_{b}$.

\section{Applications to baryon decays}

The previously introduced PWCPAs provide a systematic way to investigate the underlining dynamics of $C P \mathrm{~V}$ in multi-body decays of heavy hadrons. We propose to search for $C P \mathrm{~V}$ first through the measurements of the PWCPAs in multi-body decay channels via the transitions $b \rightarrow u \bar{u} d$ or $b \rightarrow u \bar{u} s$, for which large weak phases are expected. Candidates include $\Lambda_{b}^{0} \rightarrow p \pi^{-} \pi^{+} \pi^{-}, \Lambda_{b} \rightarrow p K^{-} \pi^{+} \pi^{-}, \Lambda_{b}^{0} \rightarrow p \pi^{-} K^{+} K^{-}, \Lambda_{b}^{0} \rightarrow \Lambda K^{+} \pi^{-}$, and $\Lambda_{b}^{0} \rightarrow p K_{s} \pi^{-}$, et al. ${ }^{4}$

\footnotetext{
${ }^{4}$ In fact, $C P V$ has been investigated in decay channels such as $\Lambda_{b}^{0} \rightarrow p \pi^{-} \pi^{+} \pi^{-}$and $\Lambda_{b} \rightarrow p K^{-} \pi^{+} \pi^{-}$, where $C P$ As associated with the triple product asymmetry $[25,26]$ were measured, and no $C P V$ was established [10, 13, 14].
} 
Take the decay channel $\Lambda_{b}^{0} \rightarrow p \pi^{-} \pi^{+} \pi^{-}$as an example. The rich resonance structure of this decay channel makes it also a perfect channel for the measurement of PWCPAs. The dominance of the resonances such as $\Delta^{++}(1232)$ and $\rho^{0}(770)$ has been observed by LHCb in this decay channel $[13,14]$. Based on a simple isospin symmetry analysis one can deduce that the resonance $\Delta^{0}(1232)$ should also dominate. Meanwhile, since the nearby resonance $N^{0}(1440)$ has a width as large as $350 \mathrm{MeV}$, the interference effect between the two resonances, $\Delta^{0}(1232)$ and $N^{0}(1440)$ (referred as $\Delta^{0}$ and $N^{0}$ respectively hereinafter), around the vicinity of $\Delta^{0}$, could be large. As a consequence, the interference between the two cascade decays, $\Lambda_{b}^{0} \rightarrow N^{0}\left(\rightarrow p \pi^{-}\right) \pi^{+} \pi^{-}$and $\Lambda_{b}^{0} \rightarrow \Delta^{0}\left(\rightarrow p \pi^{-}\right) \pi^{+} \pi^{-}$, could potentially generate PWCPAs which are large enough to be detected.

Since the spins-parities of these two resonances are $\frac{1}{2}^{+}$and $\frac{3}{2}^{+}$, respectively, from the SRs (and also, table 1) one can deduce that the non-interference terms show up for $j=0,2$, while the interference term only shows up for $j=2$. This means that the potentially large $C P \mathrm{~V}$ induced by the interference of these two nearby resonances $\Delta^{0}$ and $N^{0}$ are hence only embedded in the PWCPA $A_{C P}^{(2)}$, with no such contribution to the $\mathrm{r} C P \mathrm{~A} A_{C P}^{(0)}$ at all. The measurement of the $\mathrm{r} C P A A_{C P}^{(0)}$, as is conventionally done, would miss the interferenceinduced potentially large $C P \mathrm{~V}$. It could only be possible to find $C P \mathrm{~V}$ corresponding to the interference effect between $N^{0}$ and $\Delta^{0}$ through the measurement of $A_{C P}^{(2)}$.

To simplify the numerical estimation, while confining the invariant mass square of the $p \pi^{-}$system, $s_{p \pi^{-}}$, to be around $m_{\Delta}^{2}$, we further constrain the invariant mass of the remaining $\pi^{+} \pi^{-}$pair to be around the $\rho^{0}(770)$ (referred as $\rho^{0}$ hereinafter), which will not reduce the statistics significantly due to the dominance of $\rho^{0}$. In this situation, the decay process is dominated by two coherent cascade decays $\Lambda_{b}^{0} \rightarrow N^{0}\left(\rightarrow p \pi^{-}\right) \rho^{0}\left(\rightarrow \pi^{+} \pi^{-}\right)$and $\Lambda_{b}^{0} \rightarrow \Delta^{0}\left(\rightarrow p \pi^{-}\right) \rho^{0}\left(\rightarrow \pi^{+} \pi^{-}\right)$. Via the generalized factorization approach, the amplitudes of $\Lambda_{b}^{0} \rightarrow \Delta^{0} \rho^{0}$ and $\Lambda_{b}^{0} \rightarrow N^{0} \rho^{0}$ can both be parameterized as:

$$
\mathcal{A}_{\Lambda_{b}^{0} \rightarrow X \rho^{0}} \propto \alpha_{X}\left\langle X\left|\bar{u} \ddagger^{*}\left(1-\gamma_{5}\right) b\right| \Lambda_{b}^{0}\right\rangle,
$$

where $X=\Delta^{0}$ or $N^{0},\left\langle X\left|\bar{u} \ddagger^{*}\left(1-\gamma_{5}\right) b\right| \Lambda_{b}^{0}\right\rangle$ is the matrix element for the transition $\Lambda_{b}^{0} \rightarrow X$ with $\varepsilon$ the polarization vector of $\rho^{0}$, and

$$
\alpha_{X}=V_{u b} V_{u d}^{*} a_{2, X}-V_{t b} V_{t d}^{*} a_{4, X},
$$

with $V_{u b}, V_{u d}, V_{t b}$, and $V_{t d}$ the CKM matrix elements, $a_{i, X}=c_{i}^{\text {eff }}+c_{i-1}^{\text {eff }} / N_{C}^{\text {eff, } X}$ for $i=2,4$ $\left(c_{i}^{\text {eff }}\right.$ and $N_{C}^{\text {eff, } X}$ are the effective Wilson coefficients and color number, respectively). The amplitudes for $\Lambda_{b}^{0} \rightarrow N^{0}\left(\rightarrow p \pi^{-}\right) \rho^{0}\left(\rightarrow \pi^{+} \pi^{-}\right)$and $\Lambda_{b}^{0} \rightarrow \Delta^{0}\left(\rightarrow p \pi^{-}\right) \rho^{0}\left(\rightarrow \pi^{+} \pi^{-}\right)$can be respectively expressed in the following helicity forms:

$$
\begin{aligned}
& \mathcal{F}_{\sigma}^{\Delta} \mathcal{G}_{\lambda_{p}^{\prime}}^{\Delta} \sim \alpha_{\Delta}\left[c_{P \text {-conserve }}^{\Delta}(2 \sigma)+c_{P \text {-violate }}^{\Delta}\right]\left(2 \lambda_{p}\right) e^{i \delta_{\Delta}}, \\
& \mathcal{F}_{\sigma}^{N} \mathcal{G}_{\lambda_{p}^{\prime}}^{N} \sim \alpha_{N}\left[c_{P \text {-conserve }}^{N}+c_{P \text {-violate }}^{N}(2 \sigma)\right] e^{i \delta_{N}},
\end{aligned}
$$

where we have also written the weak decay amplitudes in the helicity form, $\mathcal{F}_{\sigma}^{\Delta / N}$, the parameters $c_{P \text {-conserve/violate }}^{\Delta, N}$ contain the form factors for the transitions $\Lambda_{b}^{0} \rightarrow \Delta^{0} / N^{0}$, the decay amplitudes for the strong decay processes $\Delta / N \rightarrow p \pi^{-}$, and some other common factors corresponding to the $\rho^{0}$ resonance which also depend on the phase space variables. 


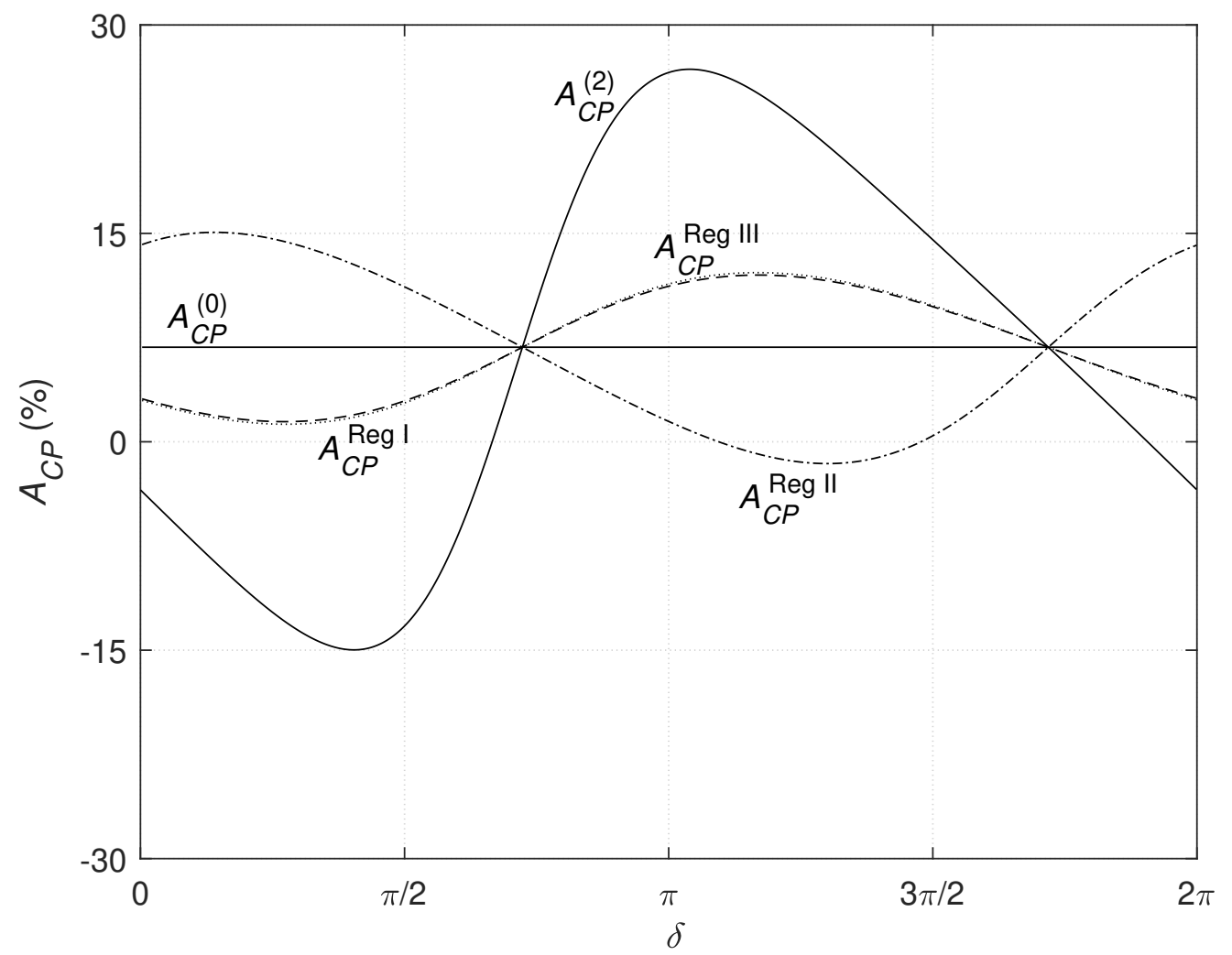

Figure 1. The PWCPA $A_{C P}^{(2)}$ (solid curvy line) for $\Lambda_{b}^{0} \rightarrow p \pi^{-} \pi^{+} \pi^{-}$near the resonance $\Delta^{0}(1232)$ as a function of the strong phase $\delta$. The regional $C P$ asymmetry $A_{C P}^{(0)}$ (solid straight line), $A_{C P}^{\text {Reg I }}$ (dotted line), $A_{C P}^{\mathrm{Reg} \text { II }}$ (dash-dotted line), and $A_{C P}^{\mathrm{Reg} \text { III }}$ (dashed line) are also shown for comparison. The difference between $A_{C P}^{\mathrm{Reg} \text { I }}$ and $A_{C P}^{\mathrm{Reg} \text { III }}$ is very tiny. Other PWCPAs $A_{C P}^{(1)}$ and $A_{C P}^{(3)}$ are not shown due to the reason explained in the text. The invariant mass squared $s_{p \pi}$ is integrated from $\left(m_{\Delta}-\Gamma_{\Delta}\right)^{2}$ to $\left(m_{\Delta}+\Gamma_{\Delta}\right)^{2}$.

Although the strong coupling constants can be extracted from the corresponding branching ratios, the weak transition form factors are not available. Moreover, the relative strong phase $\delta \equiv \delta_{N}-\delta_{\Delta}$ is not available either due to its non-perturbative nature. These prevent us from an accurate prediction for the PWCPAs in $\Lambda_{b}^{0} \rightarrow p \pi^{-} \pi^{+} \pi^{-}$. Nonetheless, for the purpose of illustrating the behaviours of the PWCPAs, we simply set all the $\left\langle c_{P-\text { conserve/violate }}^{\Delta, N}\right\rangle$ to be the same. Then, the PWCPAs are simply functions of the strong phase $\delta$, which are shown in figure $1 .^{5}$

The dependence of $A_{C P}^{(2)}$ on the strong phase $\delta$ can be clearly seen from figure 1, which is an indication of the presence of the interference term in $A_{C P}^{(2)}$, as expected. On the other hand, the $\mathrm{r} C P \mathrm{~A}, A_{C P}^{(0)}$, which is also presented in this figure for comparison, is independent of $\delta$, indicating the absence of interference term in $A_{C P}^{(0)}$, in line with the SRs constraint. Moreover, it can be seen that $A_{C P}^{(2)}$ is much larger than $A_{C P}^{(0)}$ in most regions of $\delta$, indicating

\footnotetext{
${ }^{5}$ In drawing this figure, we have used the effective Wilson's coefficients calculated according to ref. [27] with $q^{2} / m_{b}^{2}=0.3$. Besides, the effective color number is set to be $N_{C}^{\mathrm{eff}, \Delta}=2$ and $N_{C}^{\mathrm{eff}, N}=2.5$, and the CKM matrix elements are taken from ref. [28].
} 


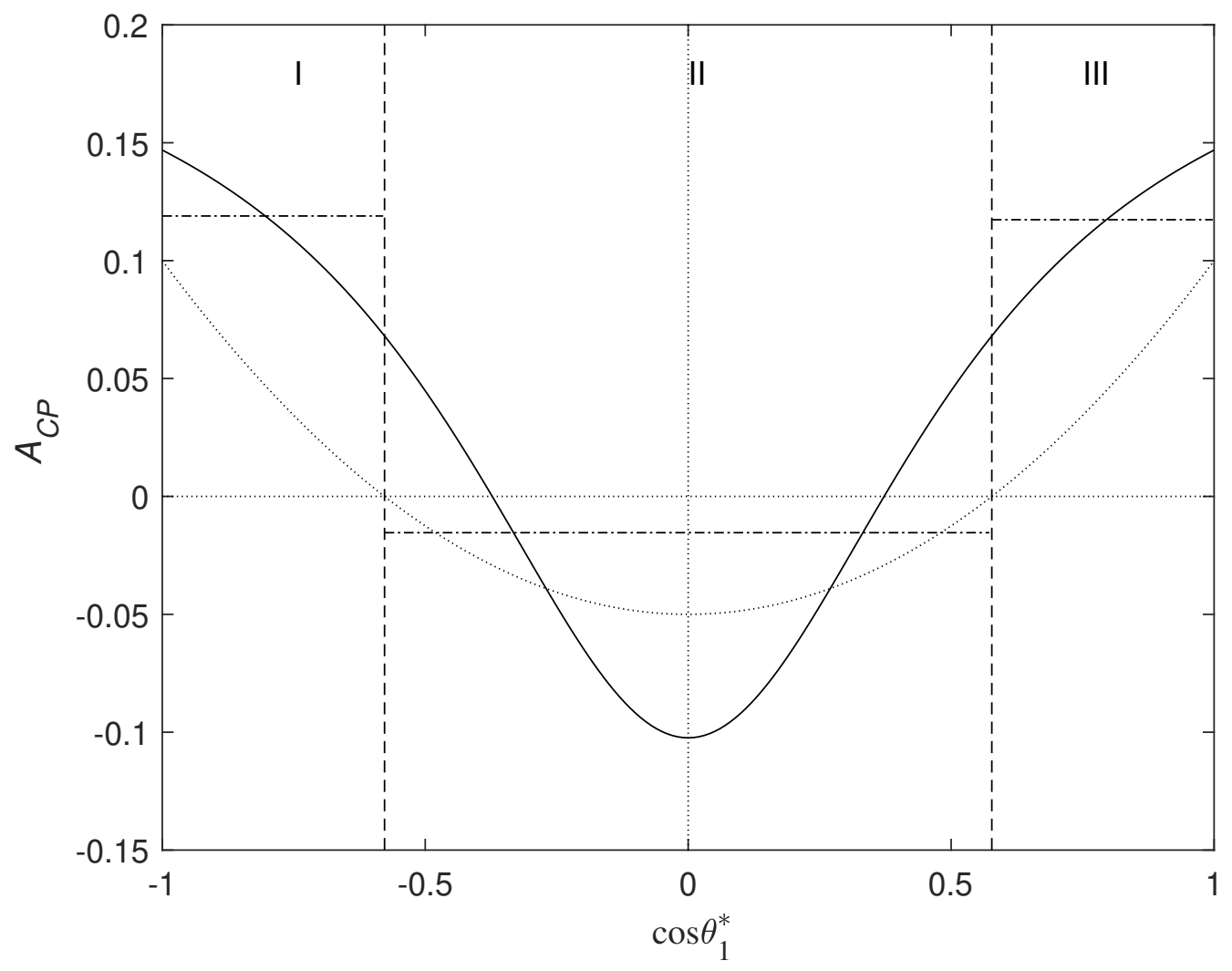

Figure 2. Differential $C P A$ for $\delta=4$ (solid line), rCPAs of the region I, II, and III (dash-dotted line in each region), are shown as a function of $c_{\theta_{1}^{*}}$. The Legendre polynomial $P_{2}$ (times 0.1 ) is also shown with dotted line.

a potentially large $C P$ asymmetry induced by the interference between $\Delta^{0}$ and $N^{0}$.

For comparison, the dependences of the differential CPAs on $c_{\theta_{1}^{*}}\left(\theta_{1}^{*}\right.$ is the angle between the proton and $\rho^{0}$ for now) for $\delta=4$ are shown in figure 2 . The whole range of $c_{\theta_{1}^{*}}$ is divided into three parts according to the sign of the Legendre polynomial $P_{2}\left(c_{\theta_{1}^{*}}\right)$, which are denoted as I, II, and III, respectively, in figure 2, corresponding to $-1<c_{\theta_{1}^{*}}<-1 / \sqrt{3}$, $-1 / \sqrt{3}<c_{\theta_{1}^{*}}<1 / \sqrt{3}$, and $1 / \sqrt{3}<c_{\theta_{1}^{*}}<1$, respectively. The $\mathrm{r} C P$ As of these three parts $A_{C P}^{\text {Reg I, II, III }}$ are also shown in this figure, from which one can see that the rCPAs tend to change signs in Region I and III comparing with the negative sign in Region II. This is a bad news for $A_{C P}^{(0)}$, because there will be cancellation between Region II and I + III. On the contrary, this is a good news for $A_{C P}^{(2)}$, as the extra $P_{2}$ in $A_{C P}^{(2)}$ results in constructive contributions from all the three parts. Indeed, the numerical values for the PWCPAs $A_{C P}^{(0)}$ and $A_{C P}^{(2)}$ are calculated to be $A_{C P}^{(0)}=6.8 \%$ and $A_{C P}^{(2)}=21.8 \%$ for $\delta=4$, respectively. In order to see more clearly, the $\delta$-dependence of the rCPAs of Region I, II, and III are also presented in figure 1, from which is can be seen that for quite a large range of the strong phase $\delta$, the PWCPA $A_{C P}^{(2)}$ is much lager than the rCPAs.

In order to illustrate the advantage of the newly introduced PWCPAs with respect to the significance and statistics, a simulation for the aforementioned decay process, $\Lambda_{b}^{0} \rightarrow$ 


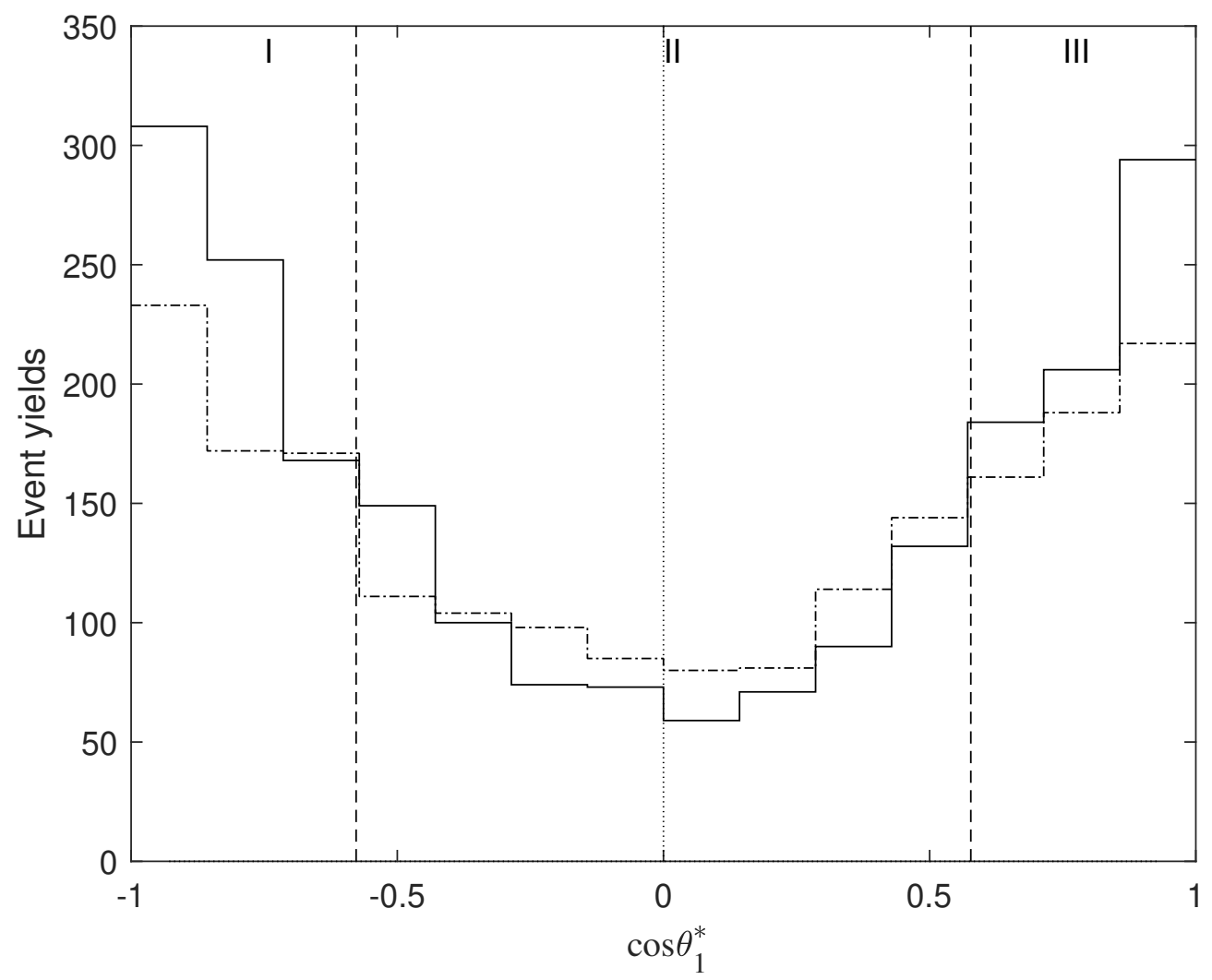

Figure 3. The simulation of the event yields for $\delta=4$. The solid line is for the baryon decay, while the dash-dotted line is for the anti-baryon decay.

$p \pi^{-} \pi^{+} \pi^{-}$, for $\delta=4$, is performed. The range of $c_{\theta_{1}^{*}}$ is uniformly divided into fourteen bins, so that the aforementioned three regions will approximately accommodate three (for Region I and III) or eight (for Region II) bins. The distributions of the simulated event yields are presented in figure 3. In total, the event yields for the baryon and the anti-baryon decay are simulated to be 2202 and 1946, respectively. ${ }^{6}$ The values and uncertainties of various $C P \mathrm{~V}$ observables calculated from the simulated data are list in table 2 , in which the uncertainties are assumed to be originated only from those of the event yields in each bin, which is estimated to be the square root of these event yields. From this table, one can see clearly that the relative uncertainty of $A_{C P}^{(2)}$ is evidently reduced comparing with those of $A_{C P}^{(0)}$ and other rCPAs. In fact, according the simulation, the significance of $A_{C P}^{(2)}$ is $6.7 \sigma$, comparing with the significance of other observables, which are $3.1 \sigma, 4.3 \sigma, 1.8 \sigma$, and $3.2 \sigma$, respectively.

Another interesting observation from figure 1 is that for certain values of $\delta$, the $A_{C P}^{(2)}$ takes values very close to zero. For example, when $\delta \approx 2.1, A_{C P}^{(2)} \approx 0$. This is mainly because the strong phase originated from the Breit-Wigner factor in the propagatror of $\Delta^{0}, 1 /\left(s_{p \pi^{-}}-m_{\Delta}^{2}+i m_{\Delta} \Gamma_{\Delta}\right)$, varies rapidly when $s_{p \pi^{-}}$is around the mass squared of $\Delta^{0}$.

\footnotetext{
${ }^{6}$ For comparison, the total signal yield for $\Lambda_{b} \rightarrow p \pi^{-} \pi^{+} \pi^{-}$are $27600 \pm 200$ according to LHCb in ref. [14]. When narrowed down to the phase space region on which we focus, our simulated event yields are reasonable. A reliable simulation of the event yield is unavailable because of various theoretical uncertainties.
} 


\begin{tabular}{|c|c|c|c|c|c|}
\hline$\delta=4$ & $A_{C P}^{(2)}$ & $A_{C P}^{(0)}$ & $A_{C P}^{\text {Reg I }}$ & $A_{C P}^{\text {Reg II }}$ & $A_{C P}^{\text {Reg III }}$ \\
\hline Simulation & $25.6 \pm 3.8$ & $4.9 \pm 1.6$ & $11.7 \pm 2.7$ & $-4.4 \pm 2.5$ & $9.0 \pm 2.8$ \\
\hline Theory & 21.8 & 6.8 & 11.9 & -1.5 & 11.7 \\
\hline
\end{tabular}

Table 2. The simulated values and uncertainties of various observables (in $\%$ ), for $\delta=4$. The theoretical values are also presented for comparison.

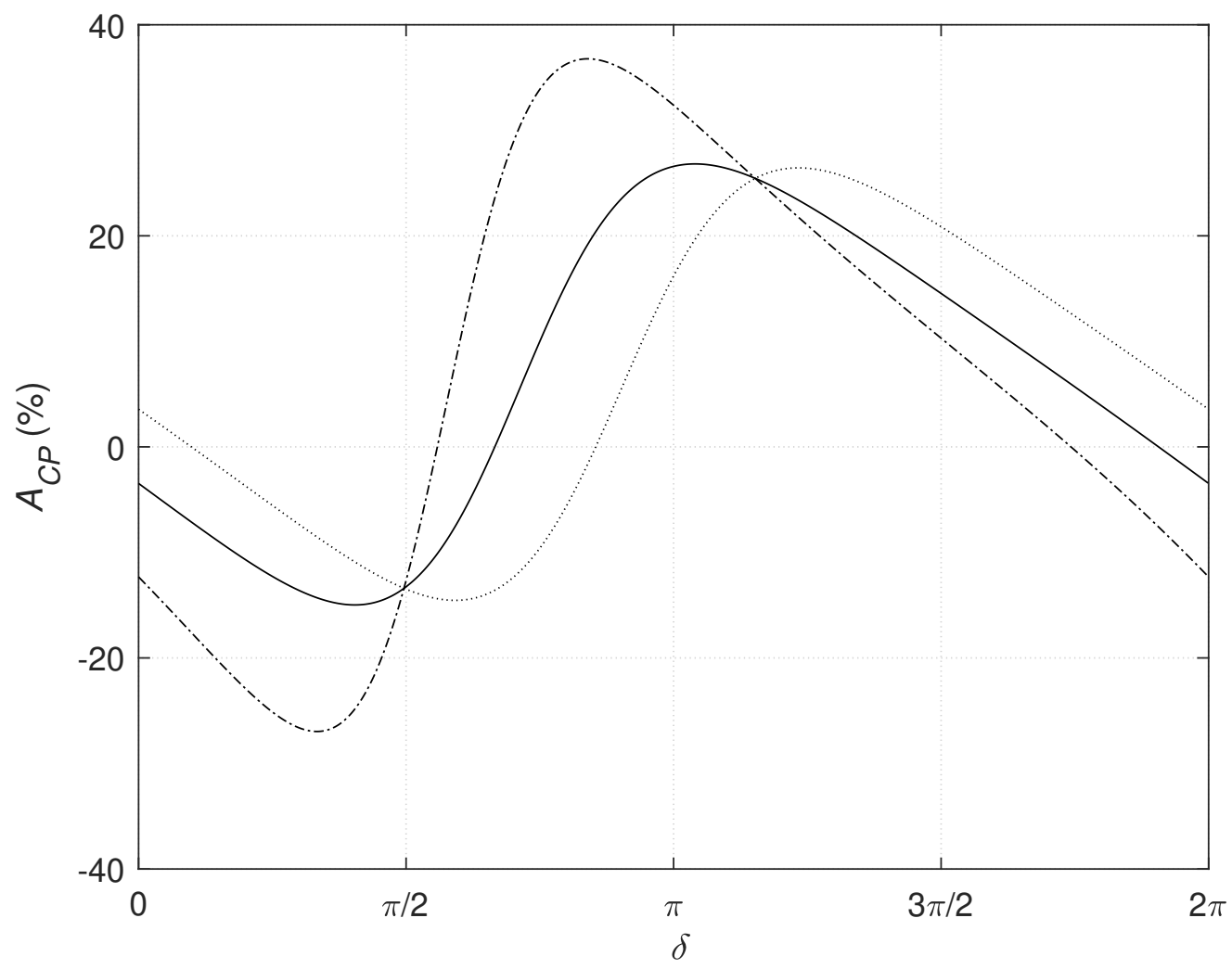

Figure 4. The PWCPAs $A_{C P}^{(2)}$ defined in different intervals of $s_{p \pi^{-}}$for $\Lambda_{b}^{0} \rightarrow p \pi^{-} \pi^{+} \pi^{-}$near the resonance $\Delta^{0}(1232)$ as a function of the strong phase $\delta$. The solid line is for the situation that $s_{p \pi^{-}}$ is integrated in the region $\left(\left(m_{\Delta}-\Gamma_{\Delta}\right)^{2},\left(m_{\Delta}+\Gamma_{\Delta}\right)^{2}\right)$, the dotted line is for that $\left(\left(m_{\Delta}-\Gamma_{\Delta}\right)^{2}, m_{\Delta}^{2}\right)$, and the dashed line is for that $\left(m_{\Delta}^{2},\left(m_{\Delta}+\Gamma_{\Delta}\right)^{2}\right)$.

As a consequence, when combined with certain values of $\delta$, large cancellation may come up, resulting in almost zero $A_{C P}^{(2)}$ when $s_{p \pi^{-}}$is integrated from $\left(m_{\Delta}-\Gamma_{\Delta}\right)^{2}$ to $\left(m_{\Delta}+\Gamma_{\Delta}\right)^{2}$. To avoid this kind of cancellation, one just needs to consider the $A_{C P}^{(2)}$ defined in a different integration interval for $s_{12}$, for example, $s_{p \pi^{-}} \in\left(\left(m_{\Delta}-\Gamma_{\Delta}\right)^{2}, m_{\Delta}^{2}\right)$, or $s_{p \pi^{-}} \in\left(m_{\Delta}^{2},\left(m_{\Delta}+\right.\right.$ $\left.\left.\Gamma_{\Delta}\right)^{2}\right)$. Indeed, a simple calculation shows that $\left.A_{C P}^{(2)}\right|_{s_{p \pi^{-}} \in\left(\left(m_{\Delta}-\Gamma_{\Delta}\right)^{2}, m_{\Delta}^{2}\right)} \approx-13.5 \%$ and $\left.A_{C P}^{(2)}\right|_{s_{p \pi^{-}} \in\left(m_{\Delta}^{2},\left(m_{\Delta}+\Gamma_{\Delta}\right)^{2}\right)} \approx 23 \%$ for $\delta=2.1$, as expected. More details about the comparison among the $A_{C P}^{(2)}$ 's defined in the aforementioned three intervals are illustrated in figure 4. On the experimental side, even if the search of $C P V$ through the measurement of PWCPAs gained nothing for the interval from $\left(m_{\Delta}-\Gamma_{\Delta}\right)^{2}$ to $\left(m_{\Delta}+\Gamma_{\Delta}\right)^{2}$, one can change this interval for another try to avoid the potentially cancellation. 


\section{Conclusion}

$C P \mathrm{~V}$ is now able to be investigated through multi-body decays of heavy hadrons such as $B$ meson, $D$ meson, bottom and charmed baryons. In view of the yet negative outcome in searching for $C P \mathrm{Vs}$ in the baryon sector, we introduce a set of new observables, the PWCPAs, which can be used to measure $C P V \mathrm{~s}$ in multi-body decays of heavy hadrons. As explained in this work, the PWCPAs provide a systematic and model-independent way in the investigation of $C P A s$ in multi-body decays of heavy hadrons. We propose to search for $C P \mathrm{Vs}$ through the measurements of the PWCPAs in multi-body decay channels of bottom and charmed baryons, such as $\Lambda_{b}^{0} \rightarrow p \pi^{-} \pi^{+} \pi^{-}, \Lambda_{b} \rightarrow p K^{-} \pi^{+} \pi^{-}, \Lambda_{b}^{0} \rightarrow p \pi^{-} K^{+} K^{-}$, $\Lambda_{b}^{0} \rightarrow \Lambda K^{+} \pi^{-}$, and $\Lambda_{b}^{0} \rightarrow p K_{s} \pi^{-}$. It is possible that $C P \mathrm{~V}$ in some multi-body decay channels of bottom and charmed baryons can be established with the strategy proposed in this paper.

\section{Acknowledgments}

We thank Prof. Wen-Bin Qian for useful discussions. This work was supported by National Natural Science Foundation of China under Contracts Nos. 11705081 and 11775024.

Open Access. This article is distributed under the terms of the Creative Commons Attribution License (CC-BY 4.0), which permits any use, distribution and reproduction in any medium, provided the original author(s) and source are credited.

\section{References}

[1] A.D. Sakharov, Violation of CP Invariance, C asymmetry, and baryon asymmetry of the universe, Pisma Zh. Eksp. Teor. Fiz. 5 (1967) 32.

[2] J.H. Christenson, J.W. Cronin, V.L. Fitch and R. Turlay, Evidence for the $2 \pi$ Decay of the $K_{2}^{0}$ Meson, Phys. Rev. Lett. 13 (1964) 138 [INSPIRE].

[3] N. Cabibbo, Unitary Symmetry and Leptonic Decays, Phys. Rev. Lett. 10 (1963) 531 [INSPIRE].

[4] M. Kobayashi and T. Maskawa, CP Violation in the Renormalizable Theory of Weak Interaction, Prog. Theor. Phys. 49 (1973) 652 [INSPIRE].

[5] BABAR collaboration, Observation of CP-violation in the $B^{0}$ meson system, Phys. Rev. Lett. 87 (2001) 091801 [hep-ex/0107013] [INSPIRE].

[6] BeLLE collaboration, Observation of large CP-violation in the neutral B meson system, Phys. Rev. Lett. 87 (2001) 091802 [hep-ex/0107061] [INSPIRE].

[7] LHCb collaboration, First observation of CP violation in the decays of $B_{s}^{0}$ mesons, Phys. Rev. Lett. 110 (2013) 221601 [arXiv:1304.6173] [INSPIRE].

[8] LHCb collaboration, Measurement of CP-violation in the phase space of $B^{ \pm} \rightarrow K^{ \pm} \pi^{+} \pi^{-}$and $B^{ \pm} \rightarrow K^{ \pm} K^{+} K^{-}$decays, Phys. Rev. Lett. 111 (2013) 101801 [arXiv:1306.1246] [INSPIRE].

[9] LHCb collaboration, Observation of CP-violation in Charm Decays, Phys. Rev. Lett. 122 (2019) 211803 [arXiv:1903.08726] [INSPIRE].

[10] LHCb collaboration, Measurement of matter-antimatter differences in beauty baryon decays, Nature Phys. 13 (2017) 391 [arXiv:1609.05216] [INSPIRE]. 
[11] LHCb collaboration, A measurement of the CP asymmetry difference in $\Lambda_{c}^{+} \rightarrow p K^{-} K^{+}$and $p \pi^{-} \pi^{+}$decays, JHEP 03 (2018) 182 [arXiv:1712.07051] [INSPIRE].

[12] LHCb collaboration, Search for $C P$ violation in $\Lambda_{b}^{0} \rightarrow p K^{-}$and $\Lambda_{b}^{0} \rightarrow p \pi^{-}$decays, Phys. Lett. B 787 (2018) 124 [arXiv:1807.06544] [INSPIRE].

[13] LHCb collaboration, Search for CP-violation using triple product asymmetries in $\Lambda_{b}^{0} \rightarrow p K^{-} \pi^{+} \pi^{-}, \Lambda_{b}^{0} \rightarrow p K^{-} K^{+} K^{-}$and $\Xi_{b}^{0} \rightarrow p K^{-} K^{-} \pi^{+}$decays, JHEP 08 (2018) 039 [arXiv: 1805.03941] [INSPIRE].

[14] LHCb collaboration, Search for CP violation and observation of $P$ violation in $\Lambda_{b}^{0} \rightarrow p \pi^{-} \pi^{+} \pi^{-}$decays, Phys. Rev. D 102 (2020) 051101 [arXiv: 1912.10741] [inSPIRE].

[15] LHCb collaboration, Search for $C P$ violation in $\Xi_{c}^{+} \rightarrow p K^{-} \pi^{+}$decays using model-independent techniques, Eur. Phys. J. C 80 (2020) 986 [arXiv:2006.03145] [InSPIRE].

[16] LHCb collaboration, Search for CP violation in $\Xi_{b}^{-} \rightarrow p K^{-} K^{-}$decays, arXiv:2104.15074 [INSPIRE].

[17] LHCb collaboration, Observation of $C P$ violation in two-body $B_{(s)}^{0}$-meson decays to charged pions and kaons, JHEP 03 (2021) 075 [arXiv:2012.05319] [INSPIRE].

[18] Y.K. Hsiao and C.Q. Geng, Direct CP-violation in $\Lambda_{b}$ decays, Phys. Rev. D 91 (2015) 116007 [arXiv: 1412.1899] [INSPIRE].

[19] I. Bediaga, I.I. Bigi, A. Gomes, G. Guerrer, J. Miranda and A.C.d. Reis, On a CP anisotropy measurement in the Dalitz plot, Phys. Rev. D 80 (2009) 096006 [arXiv:0905.4233] [INSPIRE].

[20] J.P. Dedonder, A. Furman, R. Kaminski, L. Lesniak and B. Loiseau, $S$-, $P$ - and $D$-wave final state interactions and CP-violation in $B^{ \pm} \rightarrow \pi^{ \pm} \pi^{\mp} \pi^{ \pm}$decays, Acta Phys. Polon. B 42 (2011) 2013 [arXiv: 1011.0960] [INSPIRE].

[21] Z.-H. Zhang, X.-H. Guo and Y.-D. Yang, CP violation in $B^{ \pm} \rightarrow \pi^{ \pm} \pi^{+} \pi^{-}$in the region with low invariant mass of one $\pi^{+} \pi^{-}$pair, Phys. Rev. D 87 (2013) 076007 [arXiv:1303.3676] [INSPIRE].

[22] R.H. Dalitz, On the analysis of tau-meson data and the nature of the tau-meson, Phil. Mag. 44 (1953) 1068.

[23] LHCb collaboration, Amplitude analysis of the $B^{+} \rightarrow \pi^{+} \pi^{+} \pi^{-}$decay, Phys. Rev. D 101 (2020) 012006 [arXiv: 1909.05212] [INSPIRE].

[24] M. Jacob and G.C. Wick, On the General Theory of Collisions for Particles with Spin, Annals Phys. 7 (1959) 404 [inSPIRE].

[25] M. Gronau and J.L. Rosner, Triple product asymmmetries in $\Lambda_{b}$ and $\Xi_{b}$ decays, Phys. Lett. $B 749$ (2015) 104 [arXiv:1506.01346] [InSPIRE].

[26] G. Durieux and Y. Grossman, Probing CP-violation systematically in differential distributions, Phys. Rev. D 92 (2015) 076013 [arXiv: 1508.03054] [INSPIRE].

[27] N.G. Deshpande and X.-G. He, Isospin structure of penguins and their consequences in $B$ physics, Phys. Rev. Lett. 74 (1995) 26 [Erratum ibid. 74 (1995) 4099] [hep-ph/9408404] [INSPIRE].

[28] Particle Data Group collaboration, Review of Particle Physics, PTEP 2020 (2020) 083C01 [INSPIRE]. 